\title{
A Recursive Harmony Search Algorithm for Security Constrained Transmission Expansion Planning
}

\author{
G. A. Orfanos, P. S. Georgilakis, Senior Member, IEEE, and N. D. Hatziargyriou, Fellow, IEEE \\ School of Electrical and Computer Engineering \\ National Technical University of Athens (NTUA) \\ Athens, Greece
}

\begin{abstract}
This paper proposes an enhanced recursive harmony search (HS) algorithm for the solution of the static transmission expansion planning (TEP) in deregulated environment considering security constraints. The modification of the recursive $H S$ method in comparison to the traditional HS algorithm includes: i) the introduction of a variable bandwidth in the memory consideration and the pitch adjustment phases in the improvisation step of the algorithm and ii) the possibility of restarting the algorithm after the maximum number of improvisations is reached keeping the best solutions of the previous algorithm run. In this way, the probability of finding the optimum solution of the TEP problem is highly increased without burdening the computation time of the algorithm. The proposed recursive harmony search algorithm is tested and evaluated on Garver's test system for the classic static TEP problem. Moreover, a new cost-benefit TEP formulation is introduced which is solved by the proposed HS algorithm and tested on IEEE 24-bus reliability test system considering annual system operation.
\end{abstract}

Index Terms-Harmony search, transmission expansion planning (TEP), cost-benefit ratio, security constrained optimal power flow (SCOPF).

\section{INTRODUCTION}

The deregulation of electricity markets has introduced new challenges to the transmission planners. Decisions about the expansion of the transmission system should not only be based on meeting future system reliability and security standards at a minimum investment cost but also on a cost-benefit economic analysis that takes into consideration various uncertainties associated with key market factors and provides the right signals for the optimal expansion of the generation system. The difficulty in implementing new transmission projects requires the introduction of more cost-effective and long-term planning solutions with the minimum number of new corridors in the transmission system.

The problem of classic transmission expansion planning (TEP) is a large scale, non-linear, mixed integer optimization problem that has been addressed by mathematical as well as by heuristic optimization techniques [1]. Mathematical optimization models for the TEP problem include linear programming, dynamic programming, nonlinear programming, mixed integer programming, branch and bound, Bender's decomposition, and hierarchical decomposition [1]. Heuristic models for the solution of the TEP problem include simulated annealing, greedy randomized adaptive search procedure, taboo search, genetic algorithms, differential evolution, and hybrid heuristic models [1]. The TEP problem has also been addressed by probabilistic and stochastic methods that consider random and nonrandom uncertainties in their problem formulation [2]-[4].

Transmission expansion planning in the new deregulated environment is very complex. Investment decisions should not only be based on meeting security and reliability standards by a least cost expansion plan as in classic TEP formulation, but also on the economic assessment of the impacts a new plan has on society and market. Transmission expansion can influence generation planning decisions, while demand-side management can always delay or postpone the need for network reinforcement [5]. Expansion plans should be robust and flexible against numerous uncertainties but simulation of future power system operation requires subjective judgment. Therefore, network planners have to take into consideration and examine expansion plans that guarantee security, but might be difficult to economically justify their costs, and plans whose economic justification depends critically on external parameters (e.g. fuel prices, generation planning, renewable integration, $\mathrm{CO}_{2}$ prices, etc.). Multi-objective formulation of transmission planning can successfully address some of these issues, but at the end, a compromise has to be made between security and economic aspects [6].

A meta-heuristic algorithm known as harmony search (HS) has been recently developed [7] and has been very successful in a wide variety of optimization problems, including the TEP problem [8]. The HS algorithm mimics the musicians' improvisation behavior and compared to earlier meta-heuristic optimization algorithms it imposes fewer mathematical requirements and uses a stochastic random search instead of a gradient one. Music players try to find the perfect musically pleasing harmony by improvising or "pitching" around a previous harmony, which is analogous to local and global search schemes in optimization techniques. An improved version of $\mathrm{HS}$ is presented in [9] where parameters are automatically adjusted according to its self-consciousness.

In this paper, a recursive harmony search algorithm is proposed for the solution of the TEP problem considering security constraints. The proposed algorithm can be used either for classic TEP formulation, where the objective is to find the minimum cost investments that guarantee zero load curtailment for system peak conditions, or for finding transmission investments that have the maximum benefit to cost ratio considering annual system operation under security constrained DC optimal power flow simulations (abbreviated as SC-OPF). The method is applied first to Garver six-bus test system, where the modifications of HS algorithm are 
evaluated for the classic TEP formulation. Next, the costbenefit TEP formulation is tested on IEEE 24-bus reliability test system for both reliability-driven and economic-driven investments.

\section{TEP FORMULATION}

In the classic security constrained TEP formulation, the objective is to find an optimal transmission structure and a consequent generation dispatch to meet the peak load demand with minimum investment and loss of load cost, while satisfying operational and economic limitations for normal and "N-1" contingency situations [4]. The dispatch of the generators can be considered as either fixed for normal and all the contingency situations or it can be adjusted for each of the possible network configurations [10].

In a deregulated environment a cost-benefit analysis is used to assess transmission expansion projects. For reliabilitydriven investments, whose costs are going to be socialized, the objective is to find the plan with the best "difference in social welfare" to investment cost ratio. For economic-driven investments, the objective is to find the plan with the best "congestion revenue" to investment cost ratio [11]. All economic terms should be expressed in present value. If the annual economic benefits from an investment are larger than its annualized investment cost at a selected target year, the investment is considered profitable. In case of reliabilitydriven or economic-driven investments, the objective of TEP problem is formulated by (1) or (2), respectively:

$$
\max f(x)=\frac{\Delta A S W}{A I C}=\frac{A S W_{w}-A S W_{w / o}}{A I C}
$$

or

$$
\max f(x)=\frac{A C R_{w}}{A I C}
$$

$\triangle \mathrm{ASW}$ is the difference in the annual system social welfare with $\left(\mathrm{ASW}_{\mathrm{w}}\right)$ and without $\left(\mathrm{ASW}_{\mathrm{w} / \mathrm{o}}\right)$ the proposed expansion plan (which equals the absolute annual difference in the annual congestion cost in a lossless system), $\mathrm{ACR}_{\mathrm{w}}$ is the annual congestion revenue from the proposed expansion plan and AIC is the annualized investment cost calculated by multiplying the investment cost by the capital recovery factor of the investment. In these TEP formulations, the objective is to find the minimum number of new lines that have the largest positive impact on annual system social welfare or can provide profit from the resulting price differences between the connected nodes of the new lines. Formulations (1) and (2) are suitable when a step by step search for the most profitable investments is performed, and not when the objective it to find the optimal solution of the classic or the deregulated TEP problem. In the latter case, (1) and (2) can take the following form, respectively:

$$
\max f(x)=\left(A S W_{w}-A S W_{w / o}\right)-A I C
$$

or

$$
\max f(x)=A C R_{w}-A I C
$$

The values of annual social welfare and annual congestion revenue are calculated by applying an annual security constrained OPF simulation, assuming locational marginal pricing (LMP) based electricity market. If the assumed line limits are their operational limits (total transfer capacity from each area-node to another) then a standard DC-OPF is run for each possible system configuration. On the other hand, if these limits correspond to thermal limits, then a SC-OPF is performed following [12]. Under this formulation, the $\mathrm{N}-1$ security criterion constraints are added to the original OPF constraints using LODF factors and short term emergency overload ratings, leading to a $\mathrm{N}-1$ secure final solution. For the classic TEP problem, a high penalty factor replaces the predefined value of loss load (VoLL) of the deregulated TEP problem, and only the peak load scenario is simulated by applying simple DC-OPF simulations for normal and all " $\mathrm{N}$ 1 " contingency situations.

For the reliability-driven investments, formulation (3) can provide the optimum solution in only one run of the algorithm. The annual transmission fixed cost that is added to the annual total system cost, due to the installation of the new lines, is less than the increase these new lines cause to the annual social welfare (or decrease in the annual congestion cost). Any further addition of a new line in the system will have a social welfare benefit that is less than the new line's annual cost. By using (1), for each run of the algorithm, only the best costbenefit solution is obtained, i.e. the investment plan, whose added system cost causes the higher increase in annual social welfare. The algorithm can be rebooted, using this time the new network topology with the best solution of the previous run, in order to find the new best cost-benefit solution. This procedure can continue until the algorithm cannot find any solution with a cost-benefit ratio greater than one. However, the sum of these "best" solutions may not coincide with the solution of (3), since in (1) a step by step process is followed in order to provide the planner with the plans that are the economically optimal for each (new) network topology. For the economic-driven investments, the annual cost of the new added lines must be compensated by their annual congestion revenue. The new network is not optimal in a system costbased manner, but the new lines are profitable for their owners. Similarly to the reliability-driven TEP formulation, the final solution can be obtained either in a single algorithm run by using (4) or in a step by step process using (2).

\section{Proposed Methodology}

The HS algorithm is a meta-heuristic algorithm based on an analogy with music improvisation process of searching a perfect harmony as determined by aesthetic standard. The core of the HS algorithm is the improvisation of the new harmony vector (NHV). The NHV can be the outcome of: i) random selection, ii) memory consideration and iii) memory consideration and pitch adjustment. In this paper, two new steps are added to the improved HS algorithm of [9]. The first one is added before the improvisation of the new harmony, where a variable bandwidth is being introduced in the harmony memory consideration and pitch adjustment phases. The second one is added after the end of the predefined maximum number of improvisations (NI) and helps the algorithm to overcome local optima.

The steps of the proposed HS algorithm for the solution of the aforementioned TEP problem are as follows: 
Step 1. Initialize the optimization problem and HS algorithm parameters.

The design variables of the optimization problem are the new lines that can be added in the NL predefined right of ways and the upper bound for each design variable is the maximum number of lines that can be added per right of way. The candidate new lines may also have different technical and cost characteristics. The HS algorithm parameters that have to be specified in this step are: i) the size of the harmony memory (HMS) which depends on problem's complexity, ii) the harmony memory consideration rate (HMCR), iii) the pitch adjusting rates $\left(\mathrm{PAR}_{\min }\right.$ and $\left.\mathrm{PAR}_{\max }\right)$, iv) the number of improvisations (NI) and v) the maximum number of algorithm repetitions (repetmax).

Step 2. Initialize the harmony memory (HM).

The harmony memory is initialized with randomly generated new installed lines with respect to the maximum number of lines that can be added per right of way. Each line of the HM matrix (consisting of HMS rows and NL columns) is a potential solution of TEP problem [11]. Probabilities of occurrence can be assigned to the number of new installed lines per right of way in order to obtain a less dense initial matrix. For the deregulated TEP formulation, first, the $\mathrm{ASW}_{\mathrm{w} / \mathrm{o}}$ value (annual social welfare without any expansion plans or with the best expansion plan of the previous algorithm repetition) is calculated. The candidate lines that connect nodes with high LMP differences or whose transfer capacity limit constraint is active for their branches (for either normal or N-1 capacity limit constraints) are assigned a greater probability of occurrence in the initial HM matrix. In every algorithm repetition, these probabilities are updated in order to include the corresponding results (concerning LMP differences and active capacity limit constraints) of the best expansion plan of the previous repetition

Step 3. Sort $H M$ by $f(x)$ values.

A new step before the improvisation process is introduced. The harmonies in the HM (solution vectors) are evaluated and sorted in descending order so that the solutions with higher objective function values occupy the first rows in the HM. The value of the objective function of each HM vector is calculated by applying an annual SC-OPF simulation and calculating the $f(x)$ values using one of (1), (2), (3) or (4). The number of these linear problems can be reduced by using certain load blocks of the inverse load duration curve and assuming some scenarios of availability for system generators for each load block. For each HM vector, the difference in the ASW (ACR) with and without the proposed expansion is divided by its annualized investment cost (or added to the opposite of its annualized investment cost) to form the total cost of the examined solution.

\section{Step 4. Improvise a new harmony}

A new value of a design variable in the new harmony vector (NHV) has HMCR probability to come from the HM and (1-HMCR) probability to be selected randomly (but with a certain probability) from the decision's variable initial range of values. For the memory consideration, only the first top harmonies in the sorted HM will be used for the improvisation of the new harmony vector: top $=\max \left(\operatorname{int}\left(H M S \frac{N I-j+1}{N I}\right)\right.$, topmin $) . \quad j=1,2, \ldots, N I$

This variable bandwidth allows the NHV to consider the values of the decision variables that are only at the first top harmonies of the sorted HM. In that way, the HS algorithm may use some of the previous best solutions for the improvisation step and decrease the number of improvisations (NI) needed to find the optimum solution since, as NI increases, the HS algorithm searches in a narrower bandwidth for the new value of each design variable of the NHV. To allow memory consideration until the final improvisation of the HS algorithm, top is bounded by topmin, which can be a percentage of HMS.

$$
x_{l}^{t}=y_{2} \leftarrow\left\{\begin{array}{l}
x_{l}^{t} \in\left\{x_{l}^{1}, x_{l}^{2}, \ldots, x_{l}^{\text {top }}\right\} \text { with probability HMCR } \\
x_{l}^{t} \in X_{l} \text { with probability (1-HMCR) }
\end{array}\right.
$$

Once a value of a variable has been randomly picked from the HM consideration phase, the pitch adjustment rate (PAR) determines whether further adjustment is required:

Pitch $\mathrm{y}_{2} \leftarrow\left\{\begin{array}{l}\text { Yes with probability PAR } \\ \text { No with probability (1-PAR) }\end{array}\right.$

Probability PAR is adjusted in every iteration according to the following equation:

$P A R=P A R_{\min }+\frac{j \cdot\left(P A R_{\max }-P A R_{\min }\right)}{N I} \quad j=1,2, \ldots, N I$

If the randomly generated number is less than (1-PAR) then the algorithm proceeds with the next step of the HS. If pitch adjustment decision is Yes, the decision variable is further adjusted by the following equations [5]:

$x_{l}^{t}=y_{3}=y_{2}-\left(y_{2}-\min (H M(1: \operatorname{top}))\right) \cdot \operatorname{ran}[0,1)$

or

$x_{l}^{t}=y_{4}=y_{2}+\left(\max (H M(1: \operatorname{top}))-y_{2}\right) \cdot \operatorname{ran}[0,1)$

In (7) and (8), only the first top harmonies of the HMS take part in the fine tuning of the solution vectors of the memory consideration phase.

\section{Step 5. Update the harmony memory}

The NHV is evaluated in terms of the objective function value $f(x)$. The harmony which is characterized by the lower total cost in the HM is compared to NHV total cost and if it is lower, it is replaced by the NHV.

Step 6. Repeat steps 3 to 5 until the maximum number of improvisations (NI) is reached.

Step 7. Repeat steps 2 to 6 until the maximum number of repetitions (repetmax) is reached.

The initialization process of step 2 is realized only for the last HMS-topmin harmony vectors of the sorted HM of the previous repetition of the algorithm. The first topmin rows of the HM remain the same, as they correspond to the best solutions obtained from the previous run of the algorithm. 


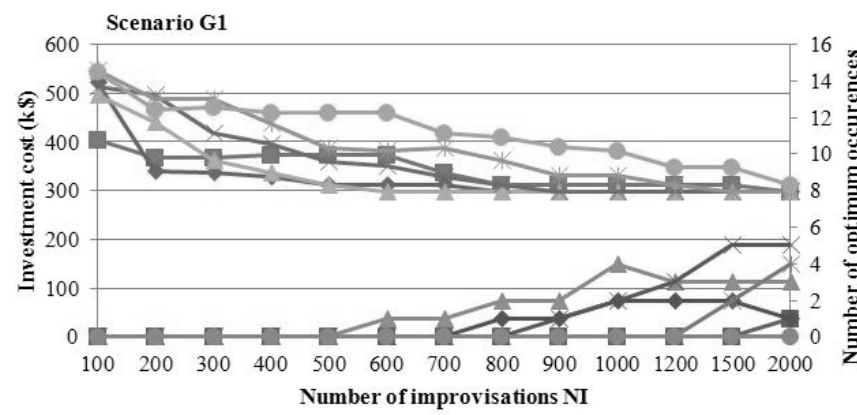

$-\mathrm{HMS}=10 \multimap \mathrm{HMS}=15 \multimap \mathrm{HMS}=25 \multimap \mathrm{HMS}=35 \multimap \mathrm{HMS}=50 \multimap \mathrm{HMS}=75$

Figure 1. Best solution of the HS algorithm and number of optimum solution occurences for various HMS and NI values after 15 runs of the algorithm for the security TEP problem.
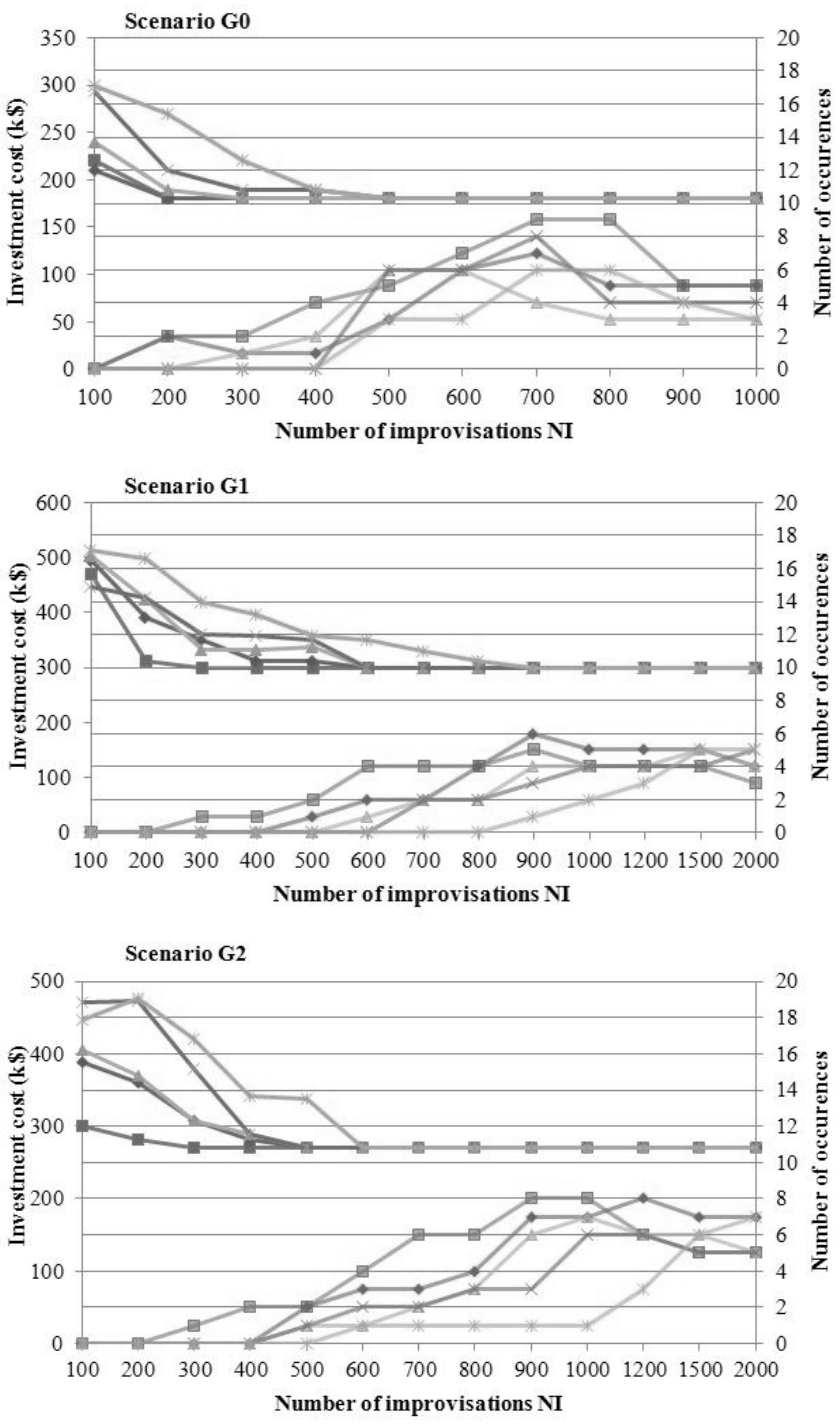

$$
\begin{aligned}
& \rightarrow \text { topmin }=0.1 \mathrm{HMS} \quad \longrightarrow \text { topmin }=0.3 \mathrm{HMS} \quad \rightarrow \text { topmin }=0.5 \mathrm{HMS} \\
& \longrightarrow \text { topmin }=0.7 \mathrm{HMS} \quad \because \text { topmin }=\mathrm{HMS}
\end{aligned}
$$

Figure 2. Best solution of the HS algorithm and number of optimum solution occurences for various topmin and NI values after 15 runs of the algorithm for the security TEP problem.

\section{RESULTS AND DISCUSSION}

\section{A. Garver's 6-Bus Test System}

The proposed recursive harmony search algorithm is first tested on Garver's 6 bus test system [13]. The performance of the algorithm is evaluated by using the classic static TEP for both re-dispatched and set generation consideration. Scenario G0 corresponds to the re-dispatched generation scenario while scenarios G1 and G2 correspond to the fixed generation examined scenarios. More specifically, for scenario G1, generation dispatch at buses 1,3 and 6 is fixed at: $\mathrm{g}_{1}=50 \mathrm{MW}$, $\mathrm{g}_{3}=165 \mathrm{MW}$ and $\mathrm{g}_{6}=545 \mathrm{MW}$ while for scenario $\mathrm{G} 2$, generation dispatch is: $g_{1}=50 \mathrm{MW}, \mathrm{g}_{3}=265 \mathrm{MW}$ and $\mathrm{g}_{6}=445 \mathrm{MW}$. The optimum solution for scenario G0 is $180 \mathrm{k} \$$, for scenario G1 is $298 \mathrm{k}$ and for scenario G2 is $270 \mathrm{k} \$$.

Initially, a sensitivity analysis around the number of improvisations NI is performed in order to find the value of parameter HMS that fits better Garver test system. In Fig. 1, the results of this sensitivity analysis for the security TEP after 15 runs of the algorithm are illustrated by using the following parameter values: $\mathrm{HMCR}=0.99, \mathrm{PAR}_{\min }=0.01, \mathrm{PAR}_{\max }=0.99$, topmin=HMS and repetmax $=1$. The best value for HMS is case dependent and follows the level of complexity of the problem. However, increasing the number of NI does not necessarily improves the probability of finding the optimum solution of the problem since parameter PAR, which is responsible for pitch adjusting the solution vectors, is directly affected by NI. In Fig. 2, the effect of the new introduced variable top on the performance of the harmony search algorithm is presented for various upper bounds topmin (as percentage of HMS) and by selecting the best value for HMS from Fig. 1, i.e. HMS=35. In all the examined scenarios, the probability of finding the optimum solution is increased compared to the topmin=HMS case for a specific value of NI. However, the algorithm can be easily trapped to local optima, especially when a small value of topmin is combined with a high value for NI.

By increasing the maximum number of repetitions (repetmax) to 15, an even better performance of the algorithm is accomplished regarding the probability of finding the optimum solution. As shown in Fig. 3, the probability of finding the optimum is very high even for a small number of NI, while the mean repetition (less or equal to repetmax) required for assuring optimum solution finding, is always reduced with increasing NI. The burden in overall computational time by using this extra step in the algorithm is very small compared to the confidence it provides in finding the optimum solution of the problem.

\section{B. IEEE 24-Bus Reliability Test System}

The proposed algorithm is also tested on IEEE 24-bus reliability test system [14]. The loads and generation levels are assumed to be three times higher than their original values and up to three lines can be installed per right of way. Parameters of candidate lines in the existing right-of-ways are the same as the parameters of the existing lines in those right-of-ways. It is assumed that the annual investment cost of transmission lines at $138 \mathrm{kV}$ is $10 \mathrm{k} \$ / \mathrm{km}$ and at $230 \mathrm{kV}$ is $20 \mathrm{k} \$ / \mathrm{km}$. The annual investment cost for each $138 / 230 \mathrm{kV}$ transformer is assumed $500 \mathrm{k}$. The optimum solution for the classic security TEP problem is $441 \mathrm{k} \$$ and the new lines added are: 

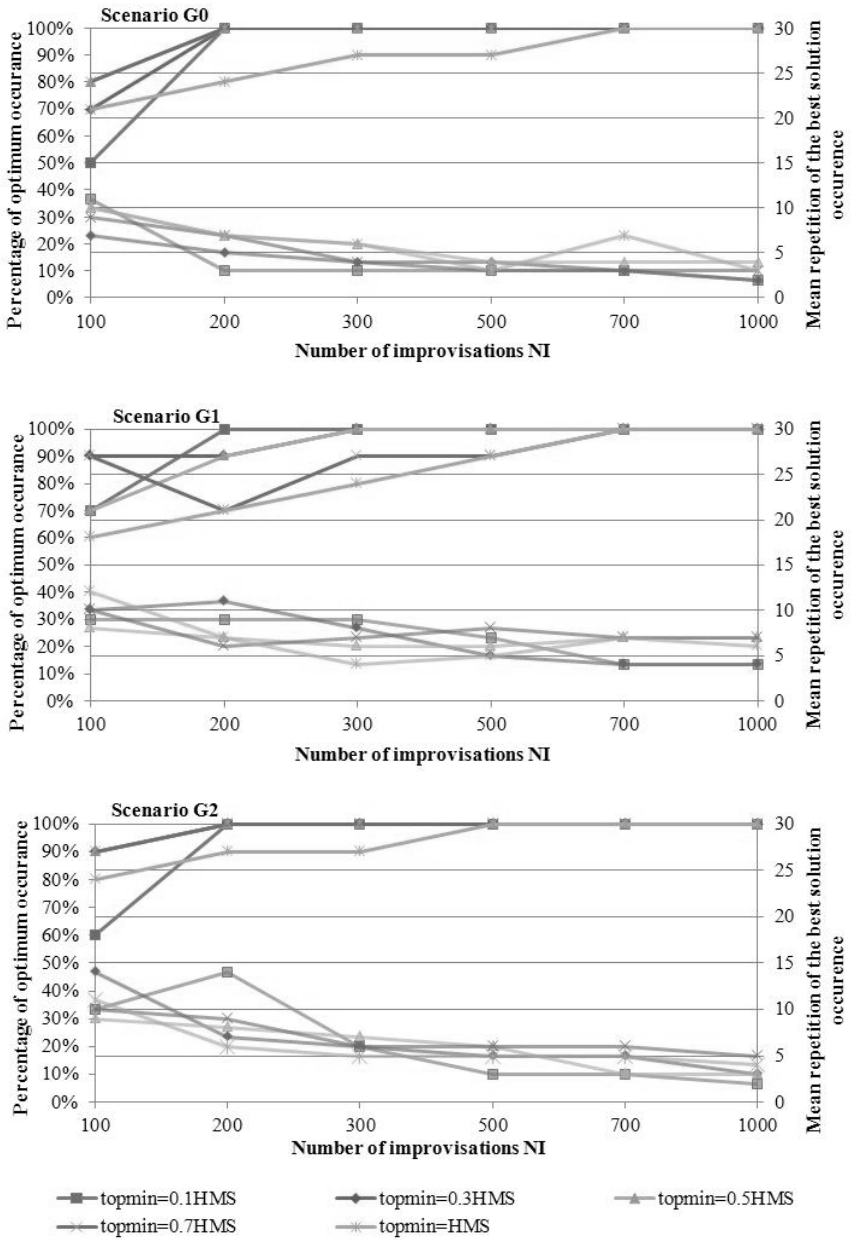

Figure 3. Percentage of finding the optimum solution and mean repetition of best solution occurence for various topmin and NI values after 15 runs of the recursive HS algorithm for the security TEP problem.

$$
\begin{aligned}
& n_{1-5}=1, n_{3-24}=1, n_{4-9}=1, n_{6-10}=2, n_{7-8}=2, \\
& n_{10-11}=1, n_{11-13}=1, n_{14-16}=1, n_{15-24}=1, n_{16-17}=1
\end{aligned}
$$

The probability of optimum solution occurrence and the mean repetitions the algorithm performed in order to find this solution for various topmin values are presented in Table I, for $\mathrm{HMS}=75, \mathrm{HMCR}=0.99$ and $\mathrm{NI}=1000$.

The improved HS algorithm is then applied to the deregulated TEP formulation, i.e. (1)-(4). Generators bid data can be found in [15] while offer price for the inelastic loads (VoLL) is set at $3000 € / M W h$. The annual power system operation is represented via eight scenarios (load blocks) whose duration and simulated load are presented in Table II. Availability of generators is assumed to be $100 \%$ for the peak load scenario 1 and drops by $5 \%$ for each subsequent scenario, thus the generators availability for scenario 8 is $65 \%$. It is assumed that the initially installed network is the one that was obtained by the solution of the classic TEP problem. Although this network configuration guarantees that there is a generation dispatch that can supply system load in normal and any single contingency situations, annual system cost (considering generation costs) is very high. The N-1 security constraints of the SCOPF problem force expensive and out of merit generators to commit throughout the year.
TABLE I. ALGORITHM PERFORMANCE FOR VARIOUS TOPMIN VALUES

\begin{tabular}{|c|c|c|c|c|c|}
\hline topmin & $\mathbf{0 . 1} \cdot \mathrm{HMS}$ & $\mathbf{0 . 3} \cdot \mathrm{HMS}$ & $\mathbf{0 . 5} \cdot \mathrm{HMS}$ & $\mathbf{0 . 7} \cdot \mathrm{HMS}$ & $\mathbf{1 . 0} \cdot \mathrm{HMS}$ \\
\hline $\begin{array}{c}\text { Probability } \\
\text { of optimum } \\
\text { occurrence }\end{array}$ & $100 \%$ & $100 \%$ & $80 \%$ & $60 \%$ & $60 \%$ \\
\hline $\begin{array}{c}\text { Mean } \\
\text { algorithm } \\
\text { repetitions }\end{array}$ & 9 & 9 & 10 & 11 & 12 \\
\hline
\end{tabular}

TABLE II. LOAD DURATION AND MEAN LOAD FOR EACH SIMULATED LOAD SCENARIO FOR THE IEEE 24-BUS RELIABILITY TEST SYSTEM

\begin{tabular}{|c|c|c|c|}
\hline $\begin{array}{c}\text { Load } \\
\text { scenario }\end{array}$ & $\begin{array}{c}\text { \% Peak } \\
\text { Load }\end{array}$ & $\begin{array}{c}\text { Duration } \\
\text { (h) }\end{array}$ & $\begin{array}{c}\text { Weighted Mean } \\
\text { Load } \\
\text { (\% Peak Load) }\end{array}$ \\
\hline$(1)$ & 100 & 2 & 100 \\
\hline$(2)$ & {$[90-100)$} & 118 & 92.51 \\
\hline$(3)$ & {$[80-90)$} & 968 & 83.85 \\
\hline$(4)$ & {$[70-80)$} & 1442 & 74.95 \\
\hline$(5)$ & {$[60-70)$} & 2034 & 64.82 \\
\hline$(6)$ & {$[50-60)$} & 1876 & 54.65 \\
\hline$(7)$ & {$[40-50)$} & 1977 & 45.50 \\
\hline$(8)$ & {$[30-40)$} & 319 & 37.80 \\
\hline
\end{tabular}

TABLE III. BEST SOLUTION OF THE RELIABILITY-DRIVEN TEP FORMULATION USING A SiNGLE RUN OF THE IMPROVED HS ALGORITHM FOR THE IEEE 24-BUS RELIABILITY TEST SYSTEM

\begin{tabular}{|c|c|c|c|c|}
\hline New lines added & $\begin{array}{c}\Delta \text { ASW } \\
(\mathbf{k \$})\end{array}$ & $\begin{array}{c}\Delta \text { ACS } \\
\mathbf{( k \$ )}\end{array}$ & $\begin{array}{c}\Delta \text { APS } \\
\mathbf{( k \$ )}\end{array}$ & $\begin{array}{c}\Delta \text { ACR } \\
(\mathbf{k} \mathbf{)}\end{array}$ \\
\hline $\begin{array}{c}\mathrm{n}_{8-9}=1, \mathrm{n}_{8-10}=1, \mathrm{n}_{11-14}=1, \\
\mathrm{n}_{13-23}=1, \mathrm{n}_{14-16}=1, \mathrm{n}_{15-21}=1, \\
\mathrm{n}_{16-17}=1, \mathrm{n}_{17-18}=2, \mathrm{n}_{21-22}=1\end{array}$ & 70,543 & 78,200 & 317,720 & $-325,377$ \\
\hline
\end{tabular}

Under formulation (3), the best solution obtained by the improved HS algorithm, using $\mathrm{HMS}=50, \mathrm{NI}=200$ and topmin $=0.3 \mathrm{HMS}$, is presented in Table III. The annual investment cost of this solution is $5,560 \mathrm{k} \$$ and the cost-benefit ratio is 12.69. In Table III, the difference in annual consumer surplus ( $\triangle \mathrm{ACS})$, annual producer surplus ( $\triangle \mathrm{APS}$ ) and annual congestion revenue $(\triangle \mathrm{ACR})$ is also demonstrated. Table IV presents the results of the HS algorithm using formulation (1). For each run of the algorithm, the calculated indices consider the final solution of the previous run. It can be observed that the two formulations do not find the same solution. This is due to the fact that although the line connecting buses 20 and 23 has the higher cost-benefit ratio at the beginning of the simulation, this line is not included in the optimum solution as presented in Table III. However, the results from the costbenefit formulation can be very useful when the transmission planner has a limited investment budget or seeks for the investment solutions that can be more easily economically justified. Uncertainties regarding generation availability (e.g. production from renewables) and bid data make the costbenefit formulation even more attractive.

If the objective of the transmission planner is to find the investment plan that will be remunerated through the collection of its annual congestion revenue, then formulations (2) and (4) should be followed. In AC transmission systems, it is very difficult to implement this kind of investment, since any line addition or reinforcement can also influence the congestion revenue of other lines while the capacity of these new lines should be used according to optimal secure and 
economic operation of the system. Nevertheless, by using (2), the most profitable expansion plans, in terms of congestion revenue to annual investment cost ratio, can be found in a step by step process. In Table V, the results of the first run of the HS algorithm for the deregulated TEP formulation (2) are presented. It should be noted that the new lines that are part of each plan may not be individually profitable.

TABLE IV. SOLUTIONS OF THE RELIABILITY-DRIVEN TEP FORMULATION USING MULTIPLE RUNS OF THE IMPROVED HS ALGORITHM FOR THE IEEE 24-BUS RELIABILITY TEST SYSTEM

\begin{tabular}{|c|c|c|c|c|}
\hline $\begin{array}{c}\text { HS } \\
\text { run }\end{array}$ & New lines added & $\begin{array}{c}\Delta \text { ASW } \\
\mathbf{( k \$ )}\end{array}$ & $\begin{array}{c}\text { AIC } \\
\mathbf{( k \$ )}\end{array}$ & $\begin{array}{c}\Delta \text { ASW/ } \\
\text { AIC }\end{array}$ \\
\hline $\mathbf{1}^{\text {st }}$ & $\mathrm{n}_{20-23}=1$ & 15,579 & 300 & 51.93 \\
\hline $2^{\text {nd }}$ & $\begin{array}{c}\mathrm{n}_{8-9}=1, \mathrm{n}_{11-14}=1, \mathrm{n}_{14-16}=1, \\
\mathrm{n}_{17-18}=1\end{array}$ & 31,391 & 1,750 & 17.94 \\
\hline $3^{\text {rd }}$ & $\begin{array}{c}\mathrm{n}_{8-10}=1, \mathrm{n}_{13-23}=1, \\
\mathrm{n}_{16-17}=1, \mathrm{n}_{17-18}=1\end{array}$ & 19,442 & 2,190 & 8.86 \\
\hline $4^{\text {th }}$ & $\mathrm{n}_{15-16}=1$ & 689 & 240 & 2.87 \\
\hline $5^{\text {th }}$ & $\begin{array}{c}\mathrm{n}_{18-21}=1, \mathrm{n}_{21-22}=1 \\
\boldsymbol{6}^{\text {th }}\end{array}$ & $\begin{array}{c}\mathbf{n}_{8-10}=1 \\
\text { Total }\end{array}$ & $\begin{array}{c}\mathrm{n}_{8-9}=1, \mathrm{n}_{8-10}=1, \\
\mathrm{n}_{11-14}=1, \mathrm{n}_{13-23}=1, \\
\mathrm{n}_{14-16}=1, \mathrm{n}_{15-16}=1, \\
\mathrm{n}_{16-17}=1, \mathrm{n}_{17-18}=2, \\
\mathrm{n}_{21-22}=1\end{array}$ \\
\hline
\end{tabular}

TABLE V. SOLUTION OF THE ECONOMIC-DRIVEN TEP FORMULATION FOR THE FIRST RUN OF THE IMPROVED HS ALGORITHM FOR THE IEEE 24-BUS RELIABILITY TEST SYSTEM

\begin{tabular}{|c|c|c|c|c|}
\hline New lines added & $\begin{array}{c}\text { AIC } \\
(\mathbf{k} \$)\end{array}$ & $\begin{array}{c}\mathbf{A C R}_{\mathbf{w}} \\
\mathbf{( k \$ )}\end{array}$ & $\begin{array}{c}\Delta \text { ASW } \\
\mathbf{( k \$ )}\end{array}$ & $\begin{array}{c}\mathbf{A C R}_{\mathbf{w}} / \\
\mathbf{A I C}\end{array}$ \\
\hline $\begin{array}{c}\mathrm{n}_{8-9}=1, \mathrm{n}_{11-14}=1, \\
\mathrm{n}_{14-16}=1, \mathrm{n}_{16-17}=1, \\
\mathrm{n}_{17-18}=1\end{array}$ & 2,110 & 37,374 & 42,103 & 17.71 \\
\hline
\end{tabular}

\section{CONCLUSIONS}

In this paper, a novel recursive harmony search algorithm has been proposed for the solution of the static transmission expansion problem with $\mathrm{N}-1$ security constraints. The new algorithm's attributes have been evaluated on Garver's test system for the classic TEP formulation. Moreover, the method was also tested on IEEE 24 bus reliability test system for both classic and deregulated TEP formulations. The results show that the probability of finding the optimal solution is very high in all the studied problems, which is a strong advantage of the proposed stochastic search method. Consequently, the proposed recursive harmony search is suitable for successfully solving the highly-complex deregulated TEP problem, which is a very time demanding and input data sensitive problem.

\section{REFERENCES}

[1] G. Latorre, R. D. Cruz, J. M. Areiza, and A. Villegas, "Classification of publications and models on transmission expansion planning," IEEE Trans. Power Syst., vol. 18, no. 2, pp. 938-946, 2003.
[2] J. Choi, T. Tran, A. A. El-Keib, R. Thomas, H. S. Oh, and R. Billinton, "A method for transmission system expansion planning considering probabilistic reliability criteria," IEEE Trans. Power Syst., vol. 20, no. 3, pp. 1606-1615, Aug. 2005.

[3] N. Yang and F. Wen, "A chance constrained programming approach to transmission system expansion planning," Electric Power Syst. Res., vol. 75, no. 2-3, pp. 171-177, 2005.

[4] G. A. Orfanos, P. S. Georgilakis, and N. D. Hatziargyriou, "Transmission expansion planning of systems with increasing wind power integration,” IEEE Trans. Power Syst., vol. 28, no. 2, pp. 13551362, May 2013.

[5] F. F. Wu, F. L. Zheng, and F. S. Wen, "Transmission investment and expansion planning in a restructured electricity market," Energy, vol.31, no. 6, pp. 954-966, May 2006.

[6] P. Maghouli, S. H. Hosseini, M. O. Buygi, and M. Shahidehpour, "A multi-objective framework for transmission expansion planning in deregulated environment,” IEEE Trans. Power Syst., vol. 24, no. 2, pp. 1051-1061, May 2009.

[7] Z. W. Geem, J. H. Kim, and G. V. Loganathan, "A new heuristic optimization algorithm: harmony search,” Simulation, vol. 76, no. 2, pp. 60-68, 2001.

[8] A. Verma, B. K. Panigrani, and P.R. Bijwe, "Harmony search algorithm for transmission network expansion planning," IET Generation, Transmission, and Distribution, vol. 4, no. 6, pp. 663-673, 2010.

[9] C. M. Wang and Y. F. Huang, "Self-adaptive harmony search algorithm for optimization,” Expert Syst. Applic., vol. 37, pp. 28262837, 2010.

[10] G. A. Orfanos, A. G. Anastopoulou, P. S. Georgilakis, and N.D. Hatziargyriou, "Static Transmission expansion planning under an improved harmony search algorithm," in Proc. IET MedPower, Cagliari, Italy, October 2012.

[11] I. I. Skoteinos, G. A. Orfanos, P. S. Georgilakis, and N. D. Hatziargyriou, "Methodology for assessing transmission investments in deregulated electricity markets,” in Proc. IEEE Trondheim PowerTech, Trondheim, Norway, June 2011.

[12] P. N. Biskas and A. G. Bakirtzis, "Decentralised security constrained DC-OPF of interconnected power systems," Proc. IEE Gener. Transm. Distrib., vol. 151, no. 6, pp. 747-754, 2004

[13] R. Villasana, L. L. Garver, and S. L. Salon, "Transmission network planning using linear programming," IEEE Trans. Power Apparatus and Systems, vol. 104, no. 2, pp. 349-356, Feb. 1985.

[14] Reliability Test System Task Force of the Application of Probability Methods Subcommittee, "IEEE Reliability Test System," IEEE Trans. Power App. Syst., vol. 98, no. 6, Nov.-Dec. 1979.

[15] G. A. Orfanos, P. S. Georgilakis, N. D. Hatziargyriou, "A more fair power flow based transmission cost allocation scheme considering maximum line loading for N-1 security,” IEEE Trans. Power Syst., in press.

George A. Orfanos was born in Athens, Greece in 1983. He received his diploma in Electrical and Computer Engineering in 2006 and his M.Eng. in Energy Production and Management in 2008, all from National Technical University of Athens (NTUA). He is currently a Ph.D. student at the School of Electrical and Computer Engineering of NTUA. His research interests include power system planning, electricity markets, and distributed generation. He is a member of the Technical Chamber of Greece.

Pavlos S. Georgilakis (M’01, SM'11) was born in Chania, Greece in 1967. He received the Diploma in Electrical and Computer Engineering and the Ph.D. degree from the National Technical University of Athens (NTUA), Athens, Greece in 1990 and 2000, respectively. He is currently Lecturer at the School of Electrical and Computer Engineering of NTUA. His current research interests include power systems optimization, renewable energy sources and distributed generation.

Nikos D. Hatziargyriou (SM'90, F'09) is Professor at the School of Electrical and Computer Engineering of NTUA. From Feb. 2007 to Sep. 2012 he was Deputy CEO of the Public Power Corporation, the Electricity Utility of Greece, responsible for Transmission and Distribution Networks. His research interests include dispersed and renewable generation, dynamic security assessment, and application of artificial intelligence techniques to power systems. Prof. Hatziargyriou is an IEEE Fellow, convener of CIGRE Study Committee C6 "Dispersed Generation” and member of the Technical Chamber of Greece 